Emergência e Medicina Intensiva

\section{ANÁLISE COMPARATIVA DOS SISTEMAS DE ASPIRAÇÄO TRAQUEAL ABERTO E FECHADO}

Jogerden IP etal., em 2007', realizaram uma metanálise com I 5 estudos randomizados, para comparar a evolução de pacientes adultos, a contaminação bacteriana e os custos da aspiração traqueal aberta (ATA) versus a aspiração traqueal fechada (ATF). Não encontraram diferença significativa na incidência de pneumonia associada à ventilação pulmonar mecânica - VPM (oito estudos) e em relação à mortalidade (quatro estudos).

Não foram possíveis conclusões sobre a saturação arterial de oxigênio - $\mathrm{SaO}_{2}$ (cinco estudos), tensão arterial de oxigênio (dois estudos) e remoção de secreções (dois estudos). Os pacientes submetidos à ATF apresentaram uma redução significativa na freqüência cardíaca [4 estudos; $R R=-6,33(I C=10,80-1,87)$ ] e na pressão arterial média [três estudos; $R R=-0,43(I C=-0,87-0,00)$ ]; entretanto, na ATF, houve um aumento na colonização bacteriana [dois estudos; $R R=1,5$ | ( $I C=1,12-2,04)$ ] e esta apresenta um custo mais elevado. Com base nesta metanálise, não existem evidências de que a ATF seja superior à ATA

\section{Comentário}

A aspiração traquealé um procedimento de rotina em unidades deterapia intensiva de pacientes adultos, pediátricos eneonatais. Tem como objetivo remover as secreções da via aérea de pacientes submetidos à VPM, permitindo uma melhor ventilação pulmonar.

NaATA, pode ocorrerestímulo do reflexo vagal (pela introdução da sonda de aspiração na via aérea além do necessário), com conseqüente diminuição da freqüência cardíaca e constrição brônquica; derrecrutamento de algumas áreas pulmonares (pela utilização em excesso de vácuo); outras complicações hemodinâmicas e ventilatórias, como a queda do volume pulmonar e da saturação arterial de oxigênio ( $\left.\mathrm{SaO}_{2}\right)$, aumento da pressão positiva final intrínseca (auto-PEEP)2. NaATF, o risco de queda da $\mathrm{SaO}_{2}$ émenor, entretanto pode ocorrer redução do volume expirado, aumento do pico de pressão inspiratória, aumento da resistência ao fluxo inspiratório e aumento da auto-PEEP durante o procedimento, dependendo do tipo de aparelho de VPM e do modo ventilatório utilizado ${ }^{3}$.

Não existem evidências sobre a supremacia entre ATA e ATF. A ATFapresentamenos efeitos adversos, mas a sua eficácia parece ser menor ${ }^{3}$. Entretanto, os efeitos adversos da ATA (queda de $\mathrm{SaO}_{2} \mathrm{e}$ derrecrutamento alveolar) podem serrevertidos pela ventilação com bolsa auto-inflável ou após o retorno do paciente ao suporte ventilatório. Para pacientes com excesso de secreção nas vias aéreas, a ATA pode ser mais eficaz 3 ,4.

Independentemente do sistema de aspiração utilizado, éimportante a monitorização dos sinais vitais e da curva pressão/volume durante e após a aspiração traqueal para minimizar os efeitos indesejados deste procedimento.

Werther Brunow de Carvalho Cíntia Johnston
Referências

I.Jongerden IP, Rovers MM, Grypdonck MH, Bonten MJ. Open and closed endotracheal suction systems in mechanically ventilated intensive care patients: A meta-analysis. Crit Care Med. 2007;35(I):260-70.

2.Lasocki S, Lu Q, Sartorius A, Fouilat D, Remerand F, Rouby JJ. Open and closed-circuit endotracheal suctioning in acute lung injury: effi-ciency and effects on gas exchange. Anesthesiology. 2006;104(1):39-47. 3.Lindgren S, Odenstedt H, Olegard C, Sondergaard S, Lundin S, Stenvist $O$. Regional lung derecruitment after endotracheal suction during volume- or pressure-controlled ventilation: a study using electric impedance tomography. Intensive Care Med. 2007;33(I):172-80.]

4.Brooks D, Anderson CM, Carter MA, et al . Clinical practice guidelines for suctioning the airway of the intubated and nonintubated patient. Can Respir J. 2006;8(2): |63-8|.

\section{Ginecalagia}

\section{ESTATINAS NA SÍNDROME DOS OVÁRIOS POLICÍSTICOS}

Em 2006, Duleba etal. ' publicaram o primeiro estudo demonstrando que a sinvastatina exerce importante ação antiandrogênica; no estudo, compararam os efeitos da sinvastatina com os de um contraceptivo (CO) antiandrogênico, ministrados durante I 2 semanas, em mulheres portadoras de síndrome de ovários policísticos e notaram queda nas concentrações séricas de testosterona em $48 \%$ contra I 4\% no grupo de usuárias de contraceptivos orais (CO); ademais, a sinvastatina diminuiu o LH (43\% versus I2\%), como também a relação LH/FSH (44\% versus I 2\%). No grupo da sinvastatina, o colesterol total declinou I 0\% e o LDL 24\%, enquanto nas usuárias de $\mathrm{CO}$ o colesterol total aumentou $10 \%$ e as concentrações de LDL séricas não se alteraram.

Em 2007, o mesmo grupo ${ }^{2}$ confirmou que a sinvastatina exibe expressivaaçãoantiandrogênica, melhorando clinicamente o hirsutismo pela queda datestosteronatotal elivre, além de diminuiro colesterol total, a LDL, a proteína C reativa e as moléculas de adesão vascular.

\section{Comentário}

Esses dois estudos - prospectivos randomizados - foram os pioneiros que testaram a hipótese de que as estatinas poderiam melhorar o hiperandrogenismo em mulheres portadoras de SOP, propiciando dessa forma uma nova abordagem que pode redundar em benefícios endócrinos e cardiovasculares. Para explicar os possíveis mecanismos envolvidos nos efeitos da sinvastatina, estudos in vitro demonstraram que as estatinas reduzem a proliferação das células tecais e intersticiais dos ovários, resultando na queda da produção de esteróides, especialmente os androgênios.

Assim, como o hiperandrogenismo se associa a fatores de risco cardiovascular, como dislipidemia e inflamação sistêmica, é possível que novos estudos com maior tamanho amostral e de longa duração venham a confirmar que as estatinas assumam uma significativa alternativa na prevenção da doença cardiovascular nas portadoras de ovários policísticos.

Ana Paula Santos Aldrighi José Mendes Aldrighi

Referências

I. Duleba AJ, Banaszewska B, Spaczynski RZ, Pawelczyk L. Simvastatin improves biochemical parameters in women with polycystic ovary syndrome: results of a prospective, randomized trial. Fertil Steril. 2006;85(4):996-100I.

2. Banaszewska B, Pawelczyk L, Spaczynski RZ, Dziura J, Duleba AJ.Effects of simvastatin and oral contraceptive agent on polycystic ovary syndrome: prospective, randomized, crossover trial. J Clin Endocrinol Metab. 2007;92(2):456-61. 